\title{
AK002, a Humanized Sialic Acid-Binding Immunoglobulin-Like Lectin-8 Antibody that Induces Antibody-Dependent Cell-Mediated Cytotoxicity against Human Eosinophils and Inhibits Mast Cell-Mediated Anaphylaxis in Mice
}

\author{
Bradford A. Youngblood ${ }^{a}$ Emily C. Brock ${ }^{a}$ John Leung ${ }^{a}$ Rustom Falahatia \\ Paul J. Bryce ${ }^{b}$ Jessica Bright ${ }^{a}$ Jason Williams ${ }^{a}$ Leonard D. Shultz ${ }^{c}$ \\ Dale L. Greiner ${ }^{d}$ Michael A. Brehm ${ }^{d}$ Christopher Bebbington ${ }^{a}$ \\ Nenad Tomasevic a \\ ${ }^{a}$ Allakos, Inc., Redwood City, CA, USA; ${ }^{b}$ Division of Allergy-Immunology, Northwestern University Feinberg School

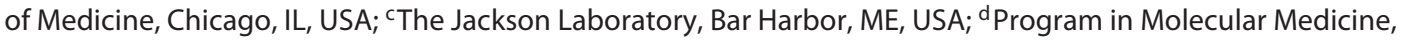 \\ University of Massachusetts Medical School, Worcester, MA, USA
}

\section{Keywords \\ AK002 - Antibody-dependent cell-mediated cytotoxicity · Anaphylaxis · Eosinophils · Mast cells $\cdot$ Sialic acid-binding immunoglobulin-like lectin-8}

\begin{abstract}
Introduction: Pathologic accumulation and activation of mast cells and eosinophils are implicated in allergic and inflammatory diseases. Sialic acid-binding immunoglobulinlike lectin (Siglec)-8 is an inhibitory receptor selectively expressed on mast cells, eosinophils and, at a lower extent, basophils. When engaged with an antibody, Siglec-8 can induce apoptosis of activated eosinophils and inhibit mast cell activation. AK002 is a humanized, non-fucosylated lgG1 anti-Siglec-8 antibody undergoing clinical investigation for treatment of allergic, inflammatory, and proliferative diseases. Here we examine the human tissue selectivity of AK002 and evaluate the in vitro, ex vivo, and in vivo activity of AKO02 on eosinophils and mast cells. Methods: The affinity of AKO02 for Siglec- 8 and CD16 was determined by biolayer interfer-
\end{abstract}

ometry. Ex vivo activity of AK002 on human eosinophils from blood and dissociated human tissue was tested in apoptosis and antibody-dependent cell-mediated cytotoxicity (ADCC) assays. The in vivo activity of a murine precursor of AK002 (mAK002) was tested in a passive systemic anaphylaxis (PSA) humanized mouse model. Results: AK002 bound selectively to mast cells, eosinophils and, at a lower level, to basophils in human blood and tissue and not to other cell types examined. AK002 induced apoptosis of interleukin-5-activated blood eosinophils and demonstrated potent ADCC activity against blood eosinophils in the presence of natural killer cells. AK002 also significantly reduced eosinophils in dissociated human lung tissue. Furthermore, mAK002 prevented PSA in humanized mice through mast cell inhibition. Conclusion: AK002 selectively evokes potent apoptotic and ADCC activity against eosinophils and prevents systemic anaphylaxis through mast cell inhibition.

(C) 2019 The Author(s)

Published by S. Karger AG, Basel

\begin{tabular}{|c|c|}
\hline KARGER & $\begin{array}{l}\text { (c) } 2019 \text { The Author(s) } \\
\text { Published by S. Karger AG, Basel }\end{array}$ \\
\hline $\begin{array}{l}\text { E-Mail karger@karger.com } \\
\text { www.karger.com/iaa }\end{array}$ & $\begin{array}{l}\text { This article is licensed under the Creative Commons Attribution- } \\
\text { NonCommercial-NoDerivatives } 4.0 \text { International License (CC BY- } \\
\text { NC-ND) (http://www.karger.com/Services/OpenAccessLicense). } \\
\text { Usage and distribution for commercial purposes as well as any dis- } \\
\text { tribution of modified material requires written permission. }\end{array}$ \\
\hline
\end{tabular}

Prof. Bradford A. Youngblood Allakos, Inc.

975 Island Drive Suite 201

Redwood City, CA 94065 (USA)

E-Mail byoungblood@allakos.com 


\section{Introduction}

Accumulation and inappropriate activation of mast cells and eosinophils are associated with pathogenesis of many allergic and inflammatory conditions. In addition to being primary effector cells in allergic responses, mast cells and eosinophils typically co-localize at sites of chronic allergic inflammation, communicating via soluble mediators and physical contact to sustain inflammation and exacerbate disease. Despite their prominent role in the pathogenesis of multiple inflammatory diseases, to date, no single therapeutic approach directly targets both cell types.

Sialic acid-binding immunoglobulin-like lectin (Siglec)-8 is an inhibitory receptor of the CD33-related subfamily of Siglecs and found only in humans and some primates [1-3]. Siglec-8 expression is restricted to mature mast cells, eosinophils and, to a much lower extent, basophils [2]. When engaged with an anti-Siglec-8 monoclonal antibody $(\mathrm{mAb})$, Siglec- 8 induces apoptosis of cytokine-activated eosinophils and inhibits mast cell activation $[4,5]$. Activation of eosinophils with pro-inflammatory cytokines sensitizes eosinophils to apoptosis-inducing effects of anti-Siglec- 8 antibodies. Several inflammatory cytokines enhance sensitivity to anti-Siglec-8-mediated killing, including interleukin (IL)-5, granulocyte-macrophage colony stimulating factor (GM-CSF), and IL-33 [5-8]. In contrast to their effect on eosinophils, anti-Siglec- $8 \mathrm{mAbs}$ do not induce apoptosis in resting or activated mast cells [4]. Instead, they inhibit IgE-mediated degranulation and de novo synthesis of prostaglandin D2 in vitro [4].

Here we characterize the selectivity of Siglec- 8 on human primary tissues and the mechanism of action of AK002, a novel humanized anti-Siglec-8 IgG1 antibody under therapeutic investigation in allergic and inflammatory diseases.

\section{Materials and Methods}

\section{Human Siglec Cross-Reactivity Enzyme-Linked}

Immunosorbent Assay

Recombinant human Siglecs $1-3,5-7,9-11$, and 14 proteins were purchased from R\&D Systems, Siglec- 4 was purchased from Sino Biological, Siglec-12 was purchased from Origene, and Siglec- 8 was produced at Allakos Inc. Maxisorp ${ }^{\mathrm{TM}}$ (Thermo Fisher Scientific) immunoassay microplates were coated with human Siglec proteins $(0.1 \mathrm{~mL}$ of $0.2 \mu \mathrm{g} / \mathrm{mL}$ for each protein diluted in Trisbuffered saline [50 mM Tris- $\mathrm{Cl}, \mathrm{pH} 7.6,150 \mathrm{~mm} \mathrm{NaCl}])$. The enzyme-linked immunosorbent assay (ELISA) plates were sealed and incubated overnight at $4^{\circ} \mathrm{C}$. The following day, plates were washed
4 times with $0.3 \mathrm{~mL}$ per well of phosphate-buffered saline (PBS) with Tween-20 (PBST; PBS [137 mM NaCl, $2.7 \mathrm{mM} \mathrm{KCl,} 10 \mathrm{mM}$ $\mathrm{Na} 2 \mathrm{HPO} 4]$ ) plus $0.1 \% \mathrm{v} / \mathrm{v}$ Tween-20. The plates were blocked with $0.3 \mathrm{~mL}$ of blocking buffer (2\% bovine serum albumin (BSA) in PBST) and placed on a shaker set to 650 revolutions per minute (RPM) for $1 \mathrm{~h}$ at room temperature. The blocking buffer was removed by washing and $0.1 \mathrm{~mL}$ of AK002 $(2 \mu \mathrm{g} / \mathrm{mL}$; Allakos, Inc.); $1 \mu \mathrm{g} / \mathrm{mL}$ mouse anti-DYKDDDDK epitope (DDK) was added to wells that contained DDK-tagged Siglec proteins in coating control wells. All other wells received the equivalent volume of blocking buffer. The plates were then incubated at room temperature for $2 \mathrm{~h}$ with shaking. The plates were then washed and secondary antibody or horseradish peroxidase (HRP) conjugates were added as follows: $0.5 \mu \mathrm{g} / \mathrm{mL}$ of goat antihuman $\mathrm{F}(\mathrm{ab}$ ')2-HRP or donkey anti-mouse (heavy and light chain $[\mathrm{H}+\mathrm{L}]$ )-HRP were added to AK002 antibody wells; $0.1 \mathrm{~mL}$ of $0.5 \mu \mathrm{g} / \mathrm{mL}$ donkey anti-human IgG $(\mathrm{H}+\mathrm{L})$-HRP was added for Fc-tagged Siglecs; $0.5 \mu \mathrm{g} / \mathrm{mL}$ of donkey anti-mouse IgG $(\mathrm{H}+\mathrm{L})$-HRP polyclonal antibody was added for mouse anti-DDK mAb wells; or $2 \mu \mathrm{g} / \mathrm{mL}$ of mouse anti-His HRP mAb was added for $6 x$ His-tagged Siglecs. The plates were then incubated for $1 \mathrm{~h}$ at room temperature with shaking. Plates were then washed with PBST and $0.1 \mathrm{~mL}$ of tetramethylbenzidine substrate (Sigma-Aldrich) was added to each well. After $2 \mathrm{~min}$, the reaction was terminated by adding $0.1 \mathrm{~mL} 1 \mathrm{M}$ sulfuric acid. The converted substrate was detected in a spectrophotometer at 450 nm.

\section{Blood and Tissue Samples}

Human blood was processed by lysing red blood cells with ammonium-chloride-potassium buffer and washed in PBS (Thermo Fisher Scientific) to obtain peripheral blood leukocytes (PBL). PBL were resuspended in RPMI 1640 medium $+10 \%$ fetal bovine serum (FBS), (Thermo Fisher Scientific). Fresh human lung and skin tissues were procured and provided by the NCI Cooperative $\mathrm{Hu}$ man Tissue Network from subjects with no previous history of chronic lung disease. Other investigators may have received specimens from the same tissue specimens. Human tissue was enzymatically and mechanically dissociated using the gentleMACs ${ }^{\mathrm{TM}}$ Dissociator (Miltenyi Biotec), according to manufacturer's protocol. Tissue was minced into $2 \mathrm{~mm}$ pieces and incubated at $37^{\circ} \mathrm{C}$ for $1 \mathrm{~h}$ in digestion solution containing proprietary enzymes. Before, during, and after incubation, tissue was mechanically disrupted and run through a 70-micron filter to obtain single cells. Cells were then treated with RBC lysing buffer, washed in PBS, and resuspended in RPMI $1640+10 \%$ Low IgG FBS. Immediately after digestion, cell viability was examined using flow cytometry. Only single-cell suspensions from dissociated tissue that had at least $70 \%$ viability were used in subsequent experiments.

Flow Cytometry Analysis in Blood and Tissue

Approximately $0.1-0.5 \times 10^{6}$ cells/well were incubated in 96well plates with Human Fc Block ${ }^{\mathrm{TM}}$ (BD Biosciences) for $10 \mathrm{~min}$ at $4{ }^{\circ} \mathrm{C}$ followed by staining with conjugated antibodies for $10 \mathrm{~min}$ at $4^{\circ} \mathrm{C}$. Cells were then washed with FACS buffer ( $0.1 \% \mathrm{BSA}$ in PBS), resuspended in fixative (1\% paraformaldehyde in PBS), and analyzed on a NovoCyte flow cytometer (Acea Biosciences). A minimum 100,000 events/sample were collected for each experiment. Antibodies and gating strategies are further described in the online supplementary Materials (for all online suppl. material, see www. karger.com/doi/10.1159/000501637).
Youngblood et al. 
Siglec-8 Receptor Density Quantification

Siglec- 8 receptor density was determined by incubating PBL purified from healthy blood with a saturating concentration of $\mathrm{Al}$ exa Fluor ${ }^{\circledR} 647$-conjugated Siglec- $8 \mathrm{mAb}$ (Allakos, Inc.) for $10 \mathrm{~min}$ on ice. PBL were co-stained with FACS antibodies against individual cell types, and populations were identified by staining and scatter as described in the supplement. Quantum Alexa Fluor ${ }^{\circledR} 647$ molecules of equivalent soluble fluorochrome (MESF) calibration standards (Bangs Laboratories, Inc.) were analyzed concurrently to allow conversion of median fluorescence intensity measurements to MESF units, according to manufacturer's protocol (Bangs Laboratories, Inc.). Background fluorescence was removed by subtracting the fluorescence minus one MESF values. MESF values were divided by the fluorophore: protein ratio (as determined by mass spectrometry) to convert to antibody binding capacity or receptor density.

Eosinophil ex vivo Depletion Assays in Human PBL or Tissue

Human PBL was prepared by removing red blood cells as described above. Human lung tissue was procured and dissociated into single cells as described above. Cells were suspended in RPMI 1640 medium containing 10\% low IgG FBS and $1 \times$ Penicillin Streptomycin (Thermo Fisher Scientific). Approximately 0.1$0.5 \times 10^{6}$ cells were plated/well in 96-well U-bottom plates with either $1 \mu \mathrm{g} / \mathrm{mL}$ AK002 or non-fucosylated hIgG1 isotype control antibodies (Allakos, Inc.) followed by overnight incubation at $37^{\circ} \mathrm{C}$. After overnight incubation, live eosinophils were identified (blood eosinophils: CD45+ 7AAD- CD16-CCR3+; tissue eosinophils: CD45+ 7AAD- SSCHi CD16- CCR3+) and quantified by flow cytometry. The percent of eosinophils remaining was calculated by normalizing the percent of CD $45+$ eosinophils in the isotype control treated wells to $100 \%$.

\section{Monovalent and Bivalent Affinity Determination}

Binding affinities of fragment antigen-binding (Fabs) and IgG for Siglec-8 extracellular domain (ECD) were measured by biolayer interferometry using a FortéBio Octet Red 384 instrument at $25^{\circ} \mathrm{C}$ at $1,000 \mathrm{rpm}$ in $1 \times$ kinetics buffer (HEPES-buffered saline; GE Healthcare) in ultrapure water, with added stabilizer (FortéBio). Fabs or IgGs were diluted from 12.5 to $0.78 \mathrm{nM}$ in assay buffer in a 2-fold dilution series. Siglec-8 ECD polyethylene glycol biotinylated protein (Allakos, Inc., San Carlos, CA, USA) was immobilized on streptavidin sensors at $100 \mathrm{nM}$ in $1 \times$ kinetics buffer for $3 \mathrm{~min}$ until a sensor change of approximately $1.8 \mathrm{~nm}$ was achieved. The association phase was 2 min followed by a $10-\mathrm{min}$ dissociation phase. An empty reference cell sensor was used as a blank control, and affinities analyzed using FortéBio analysis software with 1:1 global fit parameters. See online supplementary Materials for details.

\section{Apoptosis Assay}

Human eosinophils were purified (see online suppl. Material) and cultured overnight in $50 \mathrm{ng} / \mathrm{mL}$ of IL- 5 . Cells were then seeded in 96-well flat-bottom tissue culture plates at 50,000 eosinophils/well. AK002, AK002-G4, non-fucosylated hIgG1 isotype control, or hIgG4 isotype control antibodies (Allakos, Inc.) were added in a 10 -fold dilution series between $10 \mu \mathrm{g} / \mathrm{mL}$ and $1 \mathrm{pg} / \mathrm{mL}$ in the presence of $50 \mathrm{ng} / \mathrm{mL}$ IL-5. Cells were cultured overnight at $37^{\circ} \mathrm{C}$ in $5 \% \mathrm{CO}_{2}$. Following overnight incubation, cells were stained with 7AAD and Annexin V-PE (BD Biosciences) for 30 min at room temperature in the dark. The labeled eosinophils were analyzed by flow cytometry, and percentage of Annexin V-positive eosinophils was determined.

\section{Antibody-Dependent Cell-Mediated Cytotoxicity Assay}

Human eosinophils were purified (see online suppl. Material) and seeded in 96-well U-bottom tissue culture plates at 50,000 eosinophils/well. Purified human natural killer (NK) cells (AllCells) were washed with culture medium and added at 450,000 cells/well (effector: target cell ratio 9:1). Plates were incubated for $4 \mathrm{~h}$ at $37^{\circ} \mathrm{C}$ in $5 \% \mathrm{CO}_{2}$ for the induction of antibody-dependent cell-mediated cytotoxicity (ADCC) activity. After incubation, the plates were centrifuged at $300 \mathrm{~g}$ for $2 \mathrm{~min}$, and $50 \mu \mathrm{L}$ of supernatant was removed from each well for assay of lactate dehydrogenase to determine potential ADCC activity of the treatment antibodies. Lactate dehydrogenase assays were performed as follows: to each supernatant, $50 \mu \mathrm{L}$ CytoTox96 Assay reagent (Promega, Madison, WI, USA) was added and incubated for $30 \mathrm{~min}$ at room temperature. At the end of color development, $50 \mu \mathrm{L}$ Stop Solution (Thermo Fisher Scientific) was added, and the absorbance (optical density [OD] $495 \mathrm{~nm}$ ) was determined. As a control, $20 \mu \mathrm{L}$ of $10 \times$ cell lysis buffer (Promega) was added to an aliquot of cells to determine maximal lysis. Percent cell death was calculated for each antibody in replicate wells by dividing each sample's OD value by the OD value for $100 \%$ eosinophil lysis.

\section{Passive Systemic Anaphylaxis Model}

Passive systemic anaphylaxis (PSA) was induced using chimeric human (ch) IgE mAb as previously described [9]. NSG-SGM3 BLT mice (see online suppl. Material) were intravenously dosed with either $100 \mu \mathrm{g}$ mouse IgG1 isotype control mAb (Eureka Therapeutics) or the mouse precursor of AK002 (mAK002; Allakos, Inc.). Then, $24 \mathrm{~h}$ later NSG-SGM3 BLT mice were primed with intravenous injection of $1.6 \mu \mathrm{g}$ of ch IgE-anti-hapten 4-hydroxy-3 nitrophenacetyl (NP) antibody (Biosearch Technologies) in 200 $\mu \mathrm{L}$ and anaphylaxis was initiated $24 \mathrm{~h}$ later by intravenous injection of $500 \mu \mathrm{g}$ of NP-conjugated BSA in $100 \mu \mathrm{L}$ of PBS. Anaphylaxis was defined as a significant decrease in core body temperature and observable symptom scores as described and adapted from Ganeshan et al. [10] and Li et al. [11]. Two blinded investigators assessed symptom scores.

\section{Results}

\section{AK002 Binds Specifically to Siglec-8 and Interacts with CD16a}

AK002 is a humanized non-fucosylated IgG1 antibody with the binding specificity of the mouse anti-Siglec- 8 $\mathrm{mAb} 2 \mathrm{E} 2$ [5-7]. Using a panel of human Siglec proteins, AK002 bound specifically to the ECD of Siglec- 8 and did not show detectable cross-reactivity with other recombinant Siglec ECDs by ELISA (Fig. 1a). Kinetics for AK002 binding to recombinant Siglec-8 ECD were analyzed by biolayer interferometry. The binding affinity of a monovalent AK002 Fab was determined to be 464 pM, and the bivalent avidity of AK002 was <1 pM (Fig. 1b, c). 
To determine whether AK002 has high affinity for CD16 (Fc $\gamma$ RIII), as described for other non-fucosylated IgG1 antibodies [12, 13], CD16a and CD16b binding assays were performed by biolayer interferometry. The Fc region of AK002 had minimal interaction with immobilized CD16b, whereas the affinity of the Fc region of AK002 for immobilized CD16a (158V variant) was determined to be $9.88 \mathrm{nM}$ (online suppl. Fig. S1a, b), consistent with previous reports of the affinity of non-fucosylated IgG1 antibodies for CD16a [12-14]. In contrast, a human IgG4 version of AK002 (AK002-G4), with identical variable regions to AK002 and similar bivalent avidity to Siglec-8 ECD (online suppl. Fig. S1c), did not have detectable affinity for CD16a (online suppl. Fig. S1d).

Next, we studied binding of AK002 to CD16a and Siglec- 8 on human NK cells, T cells, eosinophils, and other immune cells from peripheral blood (online suppl. Fig. S1e). Both AK002 and AK002-G4 bound similarly to eosinophils, whereas only AK002 bound to NK cells (Fig. 1d). In contrast, no binding was observed on $\mathrm{T}$ cells, neutrophils, monocytes, or B cells, and very minimal binding was observed on basophils (Fig. 1d; online suppl. Fig. S2a). In human PBL, AK002 showed dosedependent binding to eosinophils, whereas binding to NK cells was only detectable at higher concentrations, consistent with the relative affinities of AK002 for Siglec- 8 and recombinant CD16a (Fig. 1e). Additionally, AK002-F(ab') $)_{2}$, which lacks an Fc region, bound only to eosinophils but not NK cells or T cells (online suppl. Fig. S2b). These data demonstrate that AK002 binds with high affinity to Siglec-8 in vitro, to Siglec- 8 expressed on eosinophils, and to NK cells via its Fc region in human blood.

Selective Binding of AK002 to Eosinophils in Human Peripheral Blood

To evaluate the selectivity of AK002, we used AK002G4 instead of AK002 to avoid Fc-mediated binding to CD16a-expressing cells. Human peripheral blood was analyzed using multi-color flow cytometry to identify immune cell populations that express Siglec-8 (Fig. 2a). Consistent with previously published literature using murine anti-Siglec-8 mAbs [1,2], AK002-G4 binding was observed only on eosinophils and, at much lower levels, on basophils (Fig. 2b, c). AK002-G4 did not bind blood neutrophils, monocytes, B cells, T cells, or NK cells (Fig. 2b, c; online suppl. Fig. S2c). The robust expression of Siglec- 8 on eosinophils was further confirmed using a commercially available anti-Siglec- $8 \mathrm{mAb}$ (online suppl.
Fig. S2d). Immune cells that stained positive for Siglec-8 using AK002-G4 (Fig. 2b) were further analyzed by flow cytometry to estimate Siglec-8 receptor density using the antibody-binding capacity of an anti-Siglec-8 antibody. Peripheral blood eosinophils had an average anti-Siglec- 8 binding capacity of $\sim 18,000$ per cell, compared with only $\sim 550$ per cell for basophils (Fig. 2d).

\section{Selective Binding of AK002 to Eosinophils and Mast Cells from Human Lung Tissue}

Recruitment of eosinophils from the bloodstream into tissue may be associated with altered expression of cellsurface molecules needed to sustain inflammatory and remodeling responses in the local milieu [15]. To investigate whether Siglec-8 expression was altered in tissue, we examined the binding of AK002-G4 in cells derived from dissociated human lung tissue. Using multicolor flow cytometry, we identified immune cell populations routinely found in lung, including eosinophils, mast cells, basophils, neutrophils, lymphocytes, monocytes, and macrophages (Fig. 3a). Similar to peripheral blood, AK002-G4 only bound to eosinophils and mast cells, and much less to basophils (Fig. 3b, c). AK002-G4 binding was not detected on other CD45-positive immune cells in the lung (Fig. 3b, c; online suppl. Fig. S2d, e). The robust expression of Siglec- 8 on eosinophils and mast cells was again confirmed using a commercially available anti-Siglec- 8 $\mathrm{mAb}$ (online suppl. Fig. S3a). In addition, AK002-G4 did not bind to CD45-negative cells in human lung tissue (Fig. 3d, e; online suppl. Fig. S3b). The Siglec-8-specific AK002-G4 binding profile found in human lung tissue was also consistent with the binding profile using human skin tissue (online suppl. Fig. S3c-e). These data demonstrate that AK002-G4 binding, and therefore Siglec-8 expression, is specific to eosinophils, mast cells and, to a lower extent, basophils in both human lung and skin tissues.

Tissue-derived cells that expressed Siglec- 8 (Fig. 3b) were further analyzed to quantify the receptor density of Siglec-8. Lung tissue eosinophils had an average anti-Siglec- 8 binding capacity of $\sim 21,500$ per cell and mast cells had an average binding capacity of $\sim 22,200$ per cell (Fig. 3f). As in peripheral blood, lung basophils displayed markedly lower expression of Siglec-8 than on eosinophils, with an average binding capacity of $\sim 620$ per cell (Fig. 3f). These data show that eosinophils from lung tissue have a similar number of Siglec-8 receptors as peripheral blood eosinophils, suggesting that Siglec- 8 expression is not significantly altered after migration into lung tissue.
Youngblood et al. 


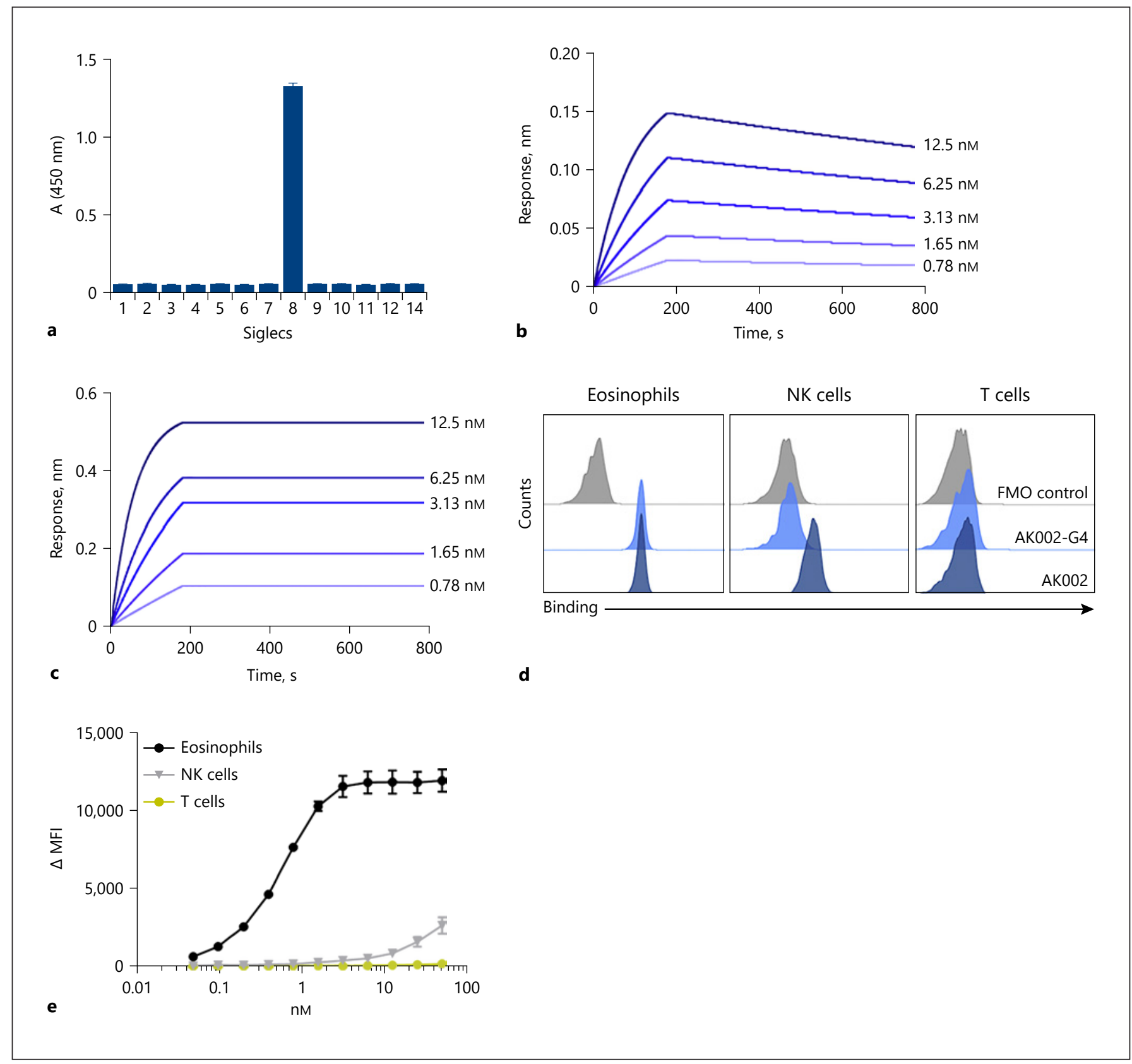

Fig. 1. AK002 is a non-fucosylated, humanized antibody that is specific for Siglec-8 and interacts with CD16a on NK cells. a AK002 specificity and cross-reactivity was examined using a recombinant human Siglec cross-reactivity ELISA. Recombinant Siglecs were coated on the plate overnight at $0.2 \mu \mathrm{g} / \mathrm{mL}$ and AK002 was added at $2 \mu \mathrm{g} / \mathrm{mL}$ for $2 \mathrm{~h}$. The binding kinetics of (b) monovalent AK002 Fabs or (c) full-length IgG AK002 (two-fold dilutions from 12.5 to $0.8 \mathrm{nM}$ ) to the Siglec- 8 ECD antigen was measured using biolayer interferometry. The following kinetic parameters were determined for the Fab and full-length IgG, respectively: $\mathrm{k}_{\text {on }}=1.04 \times 10^{6} 1 / \mathrm{Ms}$, $\mathrm{k}_{\text {off }}=4.82 \times 10^{-4} 1 / \mathrm{s}, \mathrm{Kd}=464 \mathrm{pM}$ and $\mathrm{k}_{\mathrm{on}}=1.86 \times 10^{6} 1 / \mathrm{Ms}$, since no significant dissociation was observed for the measured time of $600 \mathrm{~s} \mathrm{Kd}$ was assigned to $<1 \mathrm{pM}$. d Binding of $50 \mathrm{nM}$ AK002-G4 (light blue) and AK002 (dark blue) to eosinophils, NK cells, and T cells gated as shown in (c) in human peripheral blood by flow cytometry. e Titration of AK002 binding in human peripheral blood to eosinophils, NK cells, and $\mathrm{T}$ cells gated as shown in panel $\mathrm{C}$ by flow cytometry. Siglec, sialic acid-binding immunoglobulin-like lectin; NK, natural killer; MFI, median fluorescence intensity; FMO, fluorescence minus one. 


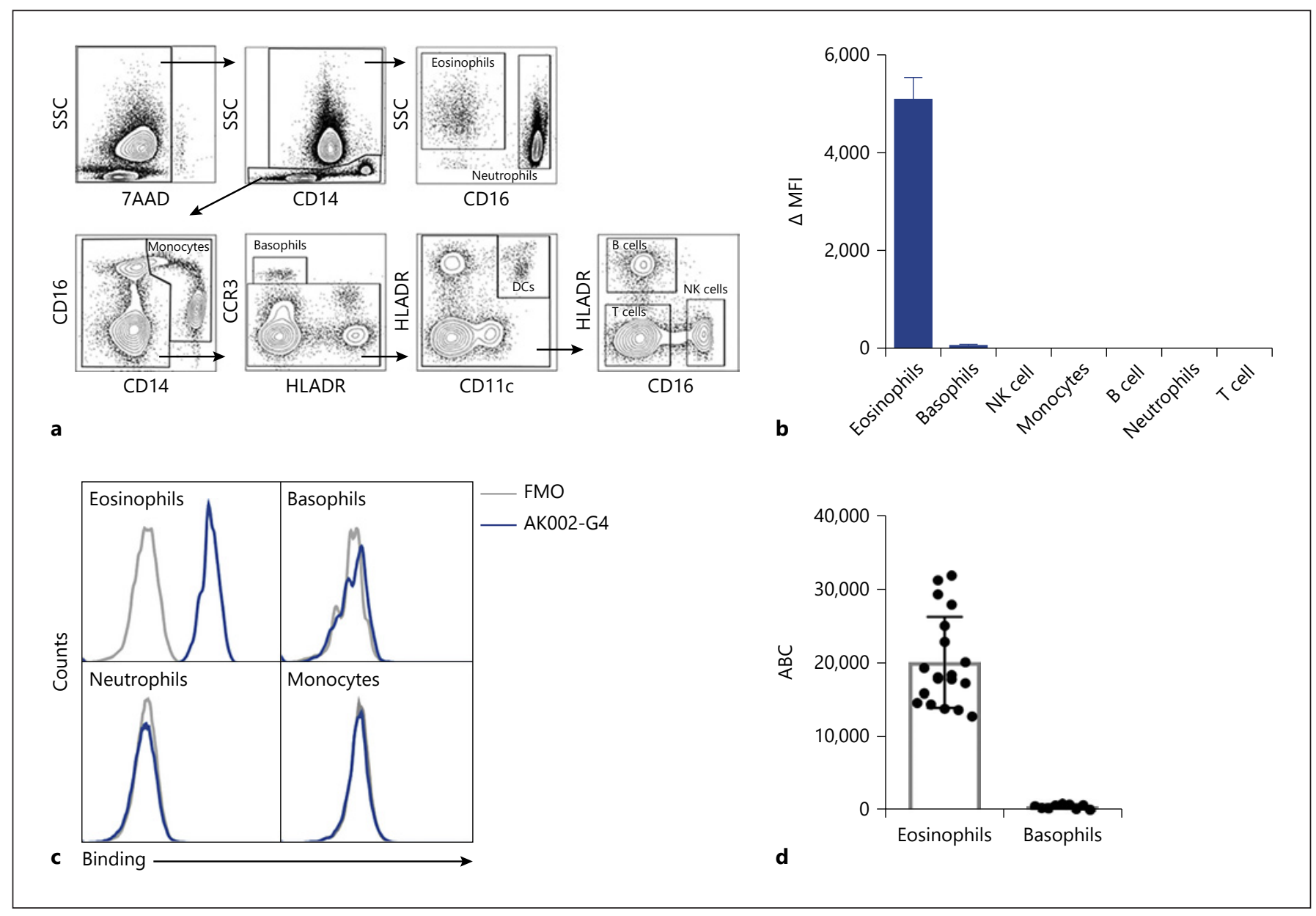

Fig. 2. AK002-G4 selectively binds to eosinophils and basophils in human peripheral blood. a Flow cytometry gating strategy used to identify immune cell subtypes among human PBL. b AK002-G4 binding plotted as $\triangle \mathrm{MFI}$ on immune cells identified using gating strategy in a (mean \pm SD of 4 donors). c Representative histograms for eosinophils, basophils, neutrophils, and monocytes stained with an FMO control (gray) or AK002-G4 (blue). d Siglec-8 ABC of peripheral blood eosinophils (median 18,029 $\pm 6,133$ ) and ba- sophils (median $552 \pm 292$ ) determined by quantitative cytometry (individual donors are plotted \pm SD). $\triangle$ MFI was determined by subtracting the MFI for a FMO control sample from the MFI for cells stained with the conjugated antibody. $\triangle \mathrm{MFI}$ values that were negative after subtracting the FMO were given a value of zero. $\mathrm{ABC}$, antibody binding capacity; MFI, median fluorescence intensity; FMO, fluorescence minus one; NK, natural killer.

duced apoptosis. In the presence of purified NK effector cells, AK002 demonstrated rapid ADCC activity against blood eosinophils (Fig. 4b). In contrast, when AK002-G4 was similarly tested in the presence of NK cells, no ADCC activity was observed (online suppl. Fig. S4b). Next, human PBL was used to examine the ADCC activity of AK002. Although IL-5 was not included in these cultures, AK002 potently depleted eosinophils in PBL preparations from healthy donors in a dose-dependent manner $\left(\mathrm{EC}_{50}=1.9 \mathrm{ng} / \mathrm{mL}\right)$, compared to isotype or AK002-G4 controls (Fig. 4c; online suppl. Fig. S4c, d), an activity consistent with ADCC-mediated killing. No reduction
Youngblood et al. 


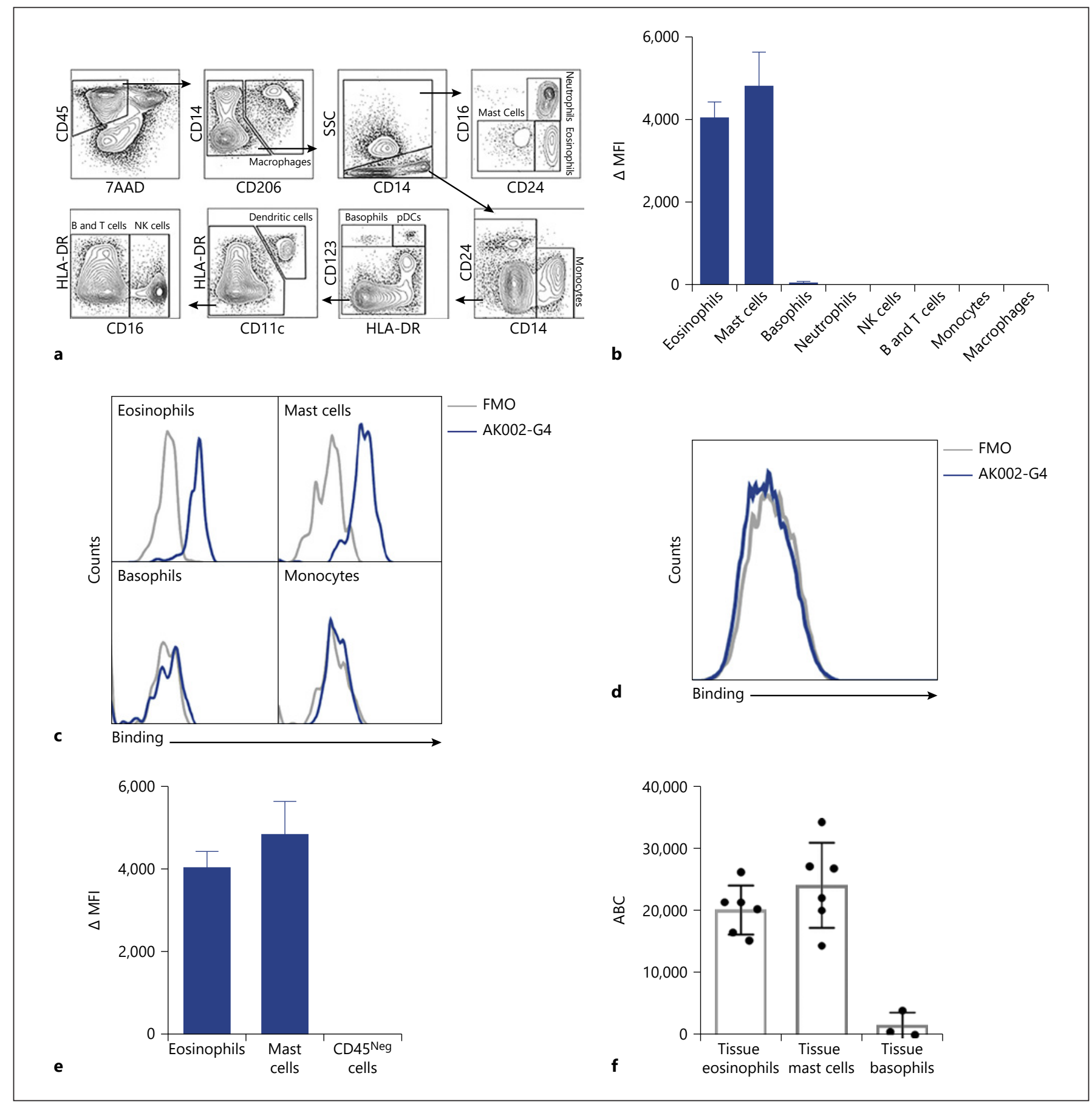

Fig. 3. AK002-G4 selectively binds to eosinophils and mast cells from human tissue. a Flow cytometry gating strategy used to identify immune cell subtypes from human lung tissue. b AK002-G4 binding plotted as $\triangle$ MFI on immune cells identified using gating strategy in (a; Mean \pm SD of 4 donors). c Representative histograms for eosinophils, mast cells, basophils, and monocytes stained with an FMO control (gray) or AK002-G4 (blue). d Representative histogram for viable CD45-negative cells in human lung tissue gated as shown above stained with an FMO control (gray) or AK002-G4 (blue). e AK002-G4 binding plotted as $\triangle \mathrm{MFI}$ on eosinophils, mast cells and viable, CD45 negative cells in human lung tissue (mean \pm SD of 4 donors). $\mathbf{f}$ Siglec- 8 ABC of lung tissue eosinophils (median $21,529 \pm 7,397$ ), lung tissue mast cells (median 22,246 $\pm 7,929$ ), and lung tissue basophils (median $621 \pm 2,085$ ) determined by quantitative cytometry (individual donors are plotted $\pm \mathrm{SD}$ ). $\triangle \mathrm{MFI}$ was determined by subtracting the MFI for an FMO control sample from the MFI for cells stained with the conjugated antibody. $\triangle \mathrm{MFI}$ values that were negative after subtracting the FMO were given a value of zero. MFI, median fluorescence intensity; FMO, fluorescence minus one; $\mathrm{NK}$, natural killer; $\mathrm{ABC}$, antibody binding capacity. 


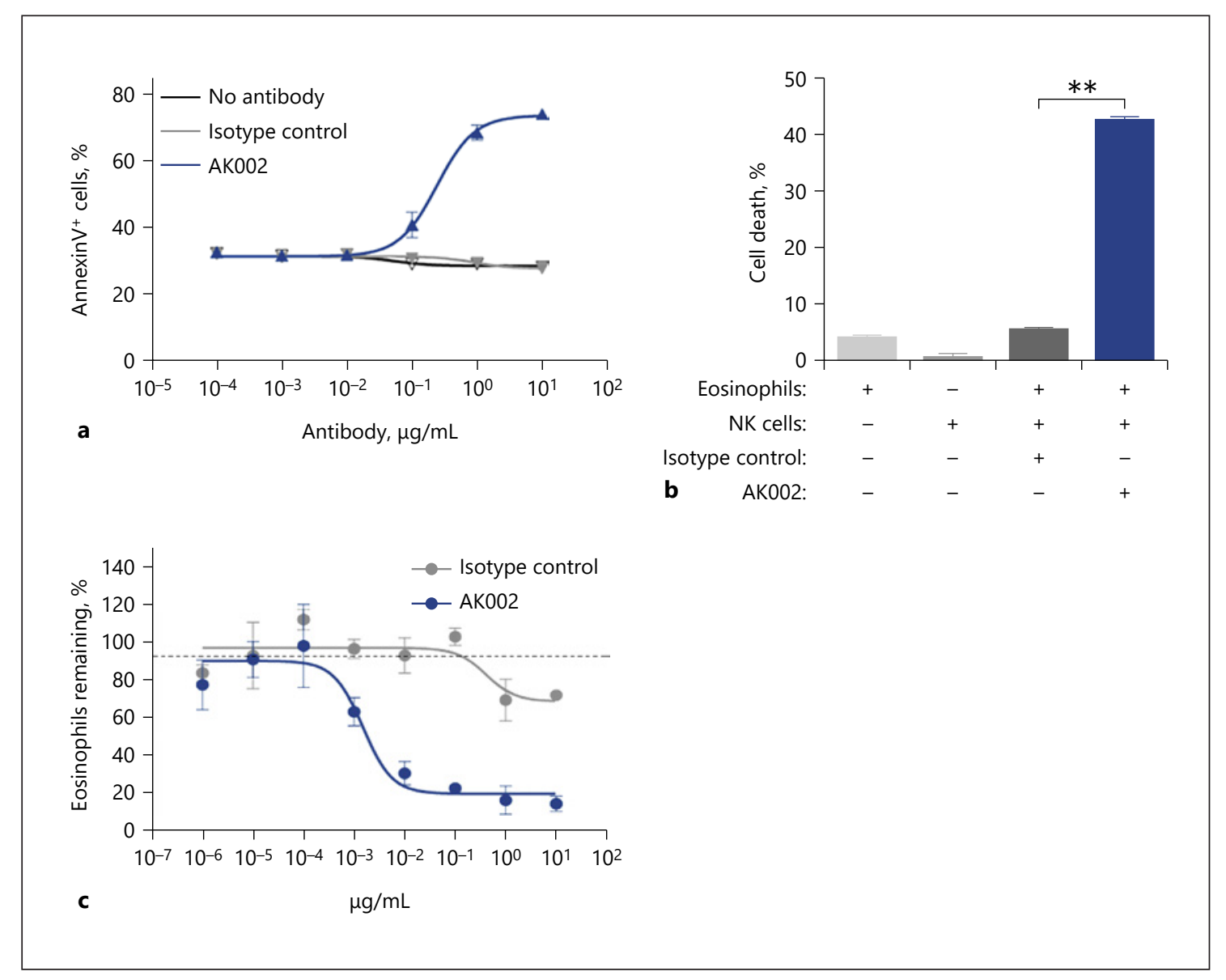

Fig. 4. AK002 has potent apoptosis-inducing activity and ADCC activity on human eosinophils. a IL-5 activated purified human eosinophils incubated overnight with AK002 (blue) or isotype control antibody (gray) followed by apoptosis detection using Annexin V-PE and 7AAD (mean \pm SD of 4 biological replicates). b Purified peripheral blood eosinophils cultured with purified NK cells at a 9:1 effector:target cell ratio in the presence of $10 \mu \mathrm{g} / \mathrm{mL}$ isotype control or AK002 for $4 \mathrm{~h}$. Cell death determined by lactate dehydrogenase release in cell supernatants (mean \pm SD of 3 bio- logical replicates). c PBL from healthy donor incubated overnight with AK002 (blue) or isotype control antibody (gray) and percentage of eosinophils remaining determined (mean \pm SD of 3 technical replicates; representative data of 3 donors). In (c), eosinophils were counted by flow cytometry and the percent eosinophils remaining was calculated by normalizing to the percent of CD45+ eosinophils in the isotype control-treated wells to $100 \% .{ }^{*} p<0.05$; ** $p<0.01$. NK, natural killer. was seen in the numbers of other immune cells found in PBL preparations (online suppl. Fig. S4e), consistent with the selective expression of Siglec-8. These data demonstrate that AK002 has dual activity on human eosinophils: direct induction of apoptosis of IL-5-activated eosinophils via Siglec- 8 and induction of potent ADCC activity against eosinophils in the presence of NK cells.

\section{AK002 Reduces Eosinophil Numbers in ex vivo \\ Human Tissue}

To begin evaluating the activity of AK002 against tissue eosinophils, we phenotyped eosinophils by flow cy- tometry to compare surface marker expression between blood and tissue eosinophils. Two cell-surface markers, CD62L and IL-5 receptor were highly expressed on peripheral blood eosinophils but were significantly downregulated on eosinophils from human lung tissue (Fig. 5a, b) [16, 17]. In contrast, Siglec-8 expression was comparable on blood and tissue eosinophils (Fig. 5a, b). AK002 significantly reduced eosinophils in ex vivo dissociated lung tissue suspensions from multiple donors compared to an isotype control antibody in the absence of exogenous cytokine (Fig. $5 c, d$ ), indicating that AK002 can reduce both blood and tissue eosinophils.
98

Int Arch Allergy Immunol 2019;180:91-102 DOI: $10.1159 / 000501637$
Youngblood et al. 


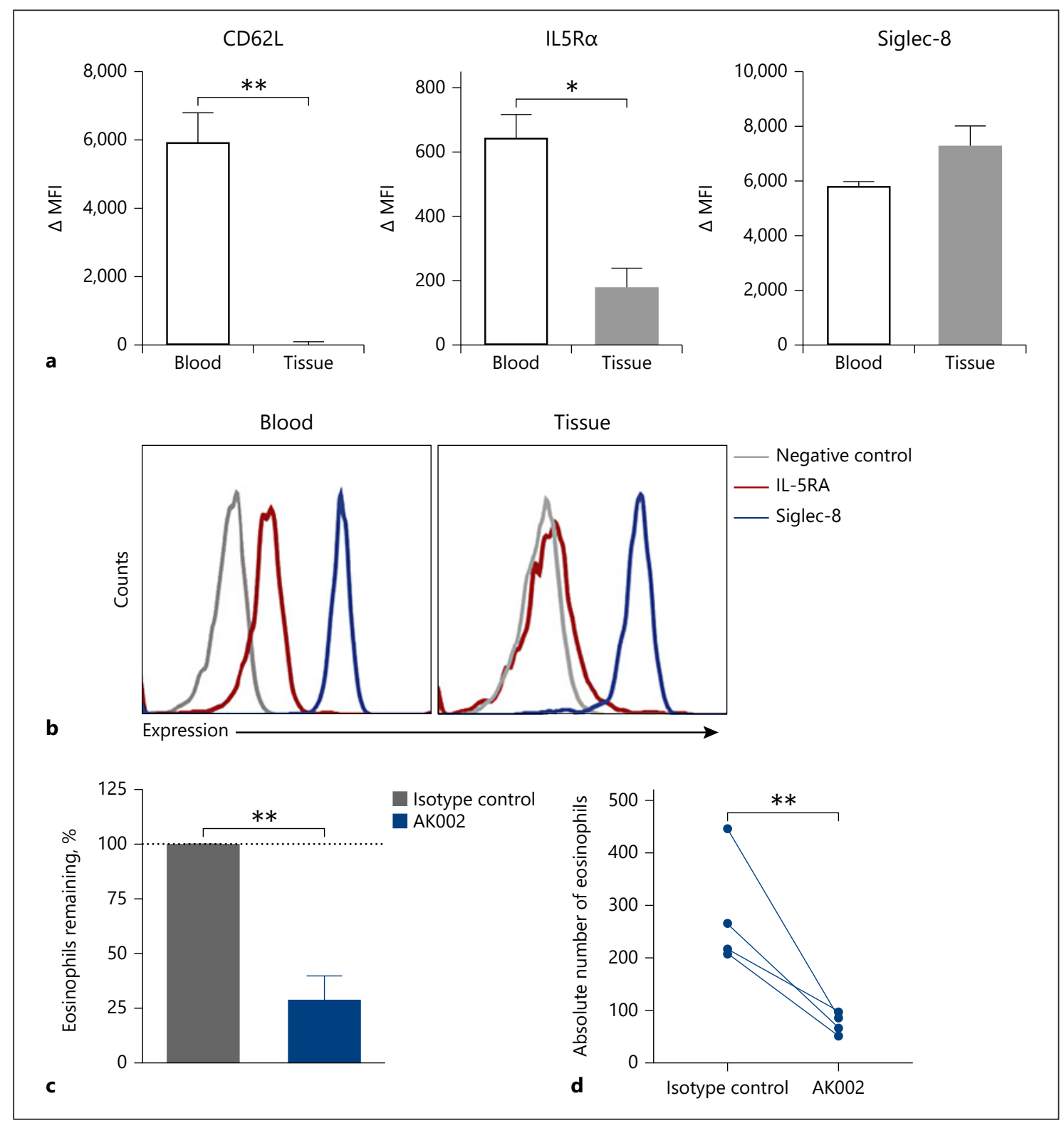

Fig. 5. AK002 reduces human tissue eosinophils ex vivo. a Surface expression of CD62L, IL-5R a and Siglec-8 plotted as $\triangle$ MFI on blood (black) and lung tissue (gray) eosinophils (mean \pm SD of 4 donors). $\mathbf{b}$ Representative histograms of surface expression of IL$5 \mathrm{Ra}$ (red), Siglec-8 (blue) or fluorescence minus one negative control (gray) on blood and lung tissue eosinophils. c, d Dissociated human tissue was incubated overnight with $1 \mu \mathrm{g} / \mathrm{mL}$ isotype control (gray) or AK002 (blue). Eosinophils were counted by flow cy-

Unlike the activity against eosinophils, AK002 did not decrease mast cell numbers in ex vivo dissociated tissue suspensions, consistent with the non-apoptosis-inducing activity of Siglec-8 on mast cells (online suppl. Fig. S4f) [4].

AK002 Depletes Human Eosinophils and Inhibits Mast Cells tometry and plotted as the (c) percent eosinophils remaining or as (d) absolute eosinophil counts. The percent eosinophils remaining was calculated by normalizing to the percent of CD $45+$ eosinophils in the isotype control-treated wells to $100 \%$ (mean \pm SD of 4 donors). ${ }^{*} p<0.05 ;{ }^{* *} p<0.01$. MFI, median fluorescence intensity; IL5R $\alpha$, interleukin-5 receptor $\alpha$; Siglec, sialic acid-binding immunoglobulin-like lectin.

\section{mAK002 Inhibits PSA in Humanized Mice}

Crosslinking of Siglec-8 on human mast cells previously has been shown to inhibit IgE-mediated mast cell activation in vitro $[4,18]$. To examine the in vivo mast cell inhibitory potential of AK002, we tested a mouse precur-

Int Arch Allergy Immunol 2019;180:91-102 


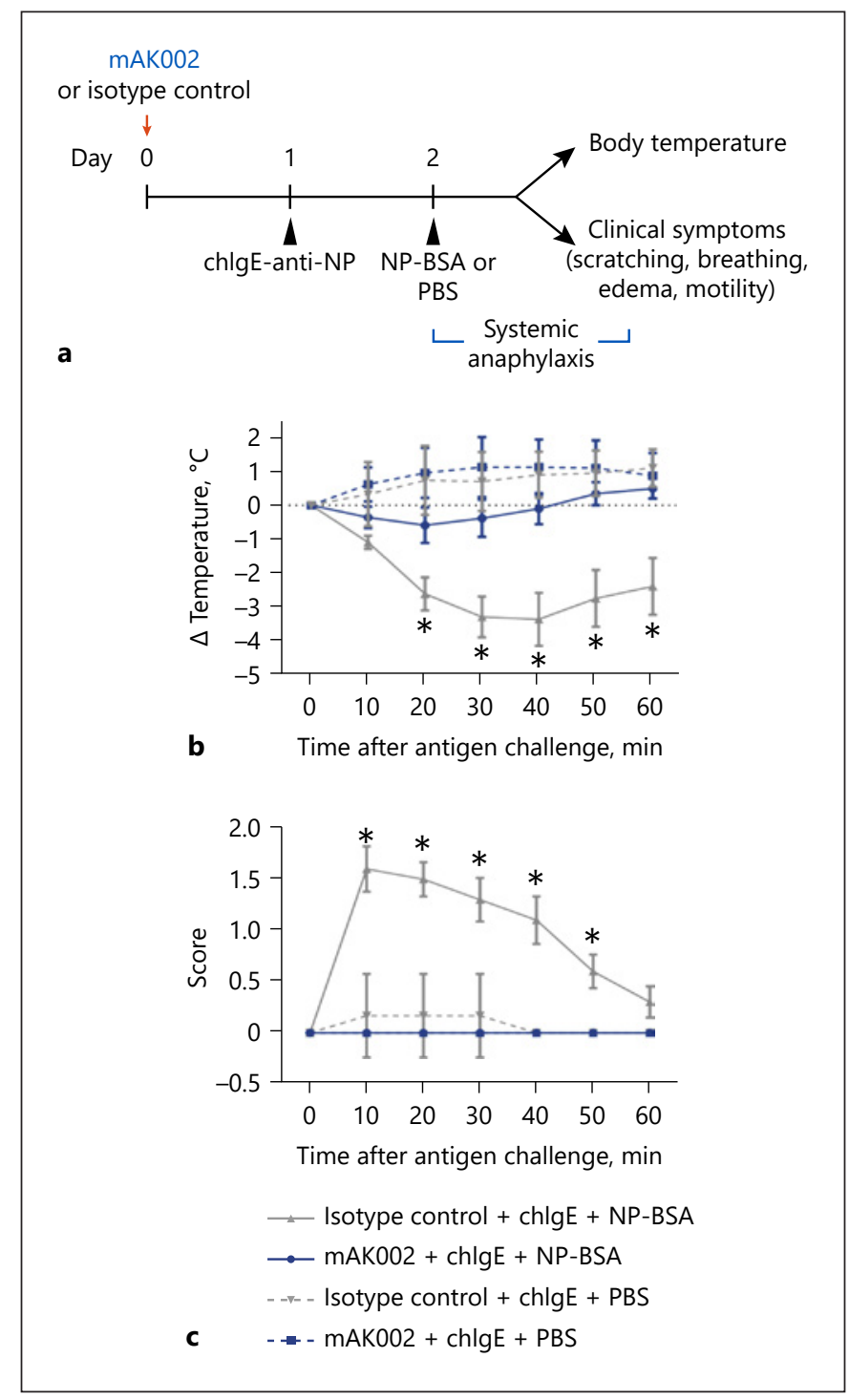

Fig. 6. mAK002 inhibits IgE-mediated PSA in humanized mice. a Experimental design of PSA in NSG-SGM3-BLT humanized mice. NSG-SGM3 BLT mice were treated with either $100 \mu \mathrm{g}$ of isotype control antibody (gray) or mAK002 (blue). The next day, the mice were systemically sensitized with chIgE-anti-NP antibody and systemically challenged $24 \mathrm{~h}$ later with BSA-NP (solid line) or PBS (dashed line). Systemic anaphylaxis was measured by monitoring the rectal body temperature $(\mathbf{b}, \mathbf{c})$ observable clinical symptom scores as described in methods (mean \pm SD of 2 experiments; $n=5-10$ mice/ group). ${ }^{*} p<0.05$. BSA, bovine serum albumin; chIgE, chimeric human IgE; NP, nitrophenacetyl; PBS, phosphate-buffered saline.

sor of AK002 (mAK002), using a humanized strain of mice (NSG-SCF/GM-CSF/IL3 (NSG-SGM3) engrafted with human thymus, liver, and HSC [BLT]), which produces mature, Siglec-8-expressing, human mast cell populations and can generate a PSA response [9]. Siglec-8 expression is restricted to mature mast cells in these mice [9]. As expected, mAK002 has the same binding affinity to Siglec-8 ECD and specificity in human blood as AK002 (online suppl. Fig. S5a, b). PSA was induced using ch IgEanti-NP and NP-BSA as antigen (Fig. 6a) [9]. NSG-SGM3 BLT mice sensitized with chIgE-anti-NP and challenged with NP-BSA, but not PBS, demonstrated systemic anaphylaxis as characterized by reduction in rectal temperature and elevated clinical symptom scores (Fig. 6b, c). mAK002, administered 24-h before IgE sensitization, completely prevented PSA as shown by a lack of change in rectal temperature and symptom scores in treated mice (Fig. 6b, c). These data demonstrate that mAK002 administration significantly inhibits IgE-mediated mast cell activation in a model of systemic anaphylaxis.

\section{Discussion/Conclusion}

Eosinophils and mast cells play pivotal roles in the pathogenesis of many allergic and inflammatory diseases. They can initiate and maintain allergic and inflammatory responses and serve as the primary effector cells leading to both acute tissue responses and long-term tissue damage [19]. Siglec- 8 is an attractive therapeutic target because of its selective expression and inhibitory activity on eosinophils and mast cells. Here we characterize AK002, a novel humanized antibody to Siglec- 8 with multiple modes of activity against eosinophils and mast cells.

We show that Siglec- 8 is expressed at high levels on the surface of eosinophils and mast cells and not on other cells in blood or tissues. Receptor quantification indicated that blood and tissue eosinophils expressed similar levels of Siglec-8 (18,000-21,500), levels comparable to those on lung mast cells $(22,000)$. Basophils showed much lower levels of Siglec-8 expression ( 600$)$ in both peripheral blood and dissociated lung tissue. The high Siglec-8 expression on blood and tissue eosinophils is consistent with recently reported levels of Siglec- 8 expression on eosinophils from peripheral blood and bronchoalveolar lavage fluid after segmental allergen challenge in subjects with allergic asthma [20]. This is in contrast to the expression of the IL-5 receptor on eosinophils, which is downregulated upon migration from the bloodstream into tissues. Siglec- 8 therefore provides an attractive opportunity for targeting both mast cells and eosinophils directly within tissues as well as eosinophils in peripheral blood.

AK002 bound selectively to Siglec- 8 with high affinity and showed no detectable binding to other Siglecs by ELISA. By flow cytometry, AK002 bound only to eosinophils 
(and modestly to basophils) in peripheral blood, and to eosinophils and mast cells (and modestly to basophils) in dissociated lung and skin tissues, showing no detectable binding to other hematopoietic or non-hematopoietic cells.

Previous studies have shown that engagement with an anti-Siglec-8 antibody induces apoptosis of cytokineprimed eosinophils mediated by $\beta 2$-integrin-dependent adhesion and generation of reactive oxygen species $[5,6$, 20]. Multiple cytokines have been shown to enhance the sensitivity of eosinophils to Siglec-8-induced apoptosis including IL-5, IL-33, GM-CSF [5-8]. Consistent with these observations, AK002 induced potent dose-dependent apoptosis of purified IL-5-activated eosinophils.

AK002 was generated as a non-fucosylated IgG1 antibody to increase its binding affinity to Fc $\gamma$ RIIIA, (CD16a) and provide enhanced NK cell-mediated ADCC activities in peripheral blood. Indeed, AK002 showed rapid induction of NK cell-mediated ADCC activity against eosinophils in vitro and potent and selective depletion of eosinophils from PBLs without a requirement for exogenous cytokines, consistent with ADCC-mediated cell depletion. The IgG4 form of AK002, which lacks ADCC function, did not induce significant depletion of eosinophils in PBL preparations in the absence of exogenous cytokine, further supporting the importance of CD16a-dependent ADCC for the activity of AK002 against peripheral blood eosinophils.

When using dissociated human lung tissues from multiple donors, AK002 significantly reduced tissue eosinophil numbers, but not mast cells ex vivo. The lack of reduction of lung tissue mast cells after AK002 is consistent with previous studies showing that Siglec-8 ligation does not induce apoptosis in mast cells [4]. These data suggest that the downstream consequences of signaling via Siglec- 8 appear to differ between eosinophils and mast cells. Siglec- 8 antibodies previously have been shown to kill eosinophils isolated from BAL from asthmatic patients by direct induction of apoptosis without the need for exogenous cytokines [6]. The ability of AK002 to directly induce apoptosis in cytokine-primed eosinophils suggests that this could be the mechanism of lung eosinophil depletion. Thus, AK002 demonstrates 2 modes of killing activity against eosinophils: NK-cell mediated ADCC activity in peripheral blood and direct induction of apoptosis of tissue eosinophils.

The lack of mast cell depletion by AK002 in human lung tissue suggests that ADCC is not highly active in tissue. In support of this, ADCC has been shown to be mediated by NK cells of the $\mathrm{CD} 16^{\mathrm{Hi}}$ phenotype which are most abundant in the blood [21,22]. NK cells found in tissue are rare, and even when present are thought to have a CD $16^{\mathrm{Lo}}, \mathrm{CD} 56^{\mathrm{Hi}}$ phenotype with reduced ADCC activ-

AK002 Depletes Human Eosinophils and Inhibits Mast Cells ity [21]. These data suggest that ADCC against eosinophils mainly occurs in the blood rather than tissue due to differences in number and function of local NK cell populations. Despite the lack of ADCC-inducing NK cells in lung tissue, AK002 significantly depleted tissue eosinophils, likely via other mechanisms of anti-eosinophilic activity such as apoptosis.

In addition to the depleting activity against eosinophils, anti-Siglec- 8 antibodies previously have been shown to inhibit mast cell activation in vitro $[4,18]$. To examine if antibody engagement of Siglec- 8 can inhibit IgE-mediated mast cell activation in vivo, we used a PSA mouse model which produces human mast cells that populate the peripheral immune system and mucosal tissues [11]. Human mast cells in NSG-SGM3 BLT mice are phenotypically similar to primary human mast cells, expressing Siglec-8, CD117, tryptase, and IgE receptor [9]. These mice developed a human IgE-mediated PSA response following systemic challenge with NP-BSA that was completely prevented by treatment with $\mathrm{mAK002}$. As a result, the dual mechanism of action of AK002 against eosinophils, along with its inhibition of mast cell activation, represents a promising approach for the treatment of allergic and inflammatory diseases.

In summary, we report that Siglec- 8 is robustly and selectively expressed on eosinophils and mast cells in human blood and tissue. AK002, a humanized non-fucosylated IgG1 anti-Siglec-8 antibody, selectively binds to human eosinophils, mast cells and, to a lesser extent, basophils, consistent with the restricted expression of Siglec-8. AK002 has potent ADCC and apoptosis-inducing activity against peripheral blood eosinophils and human tissue eosinophils. The antibody also shows inhibitory activity against mast cells in vivo. These findings support the therapeutic potential of AK002 for the treatment of eosinophil and mast cell-related diseases and, pursuant to this, studies are currently underway in mast cell and eosinophilic diseases (eosinophilic gastritis and gastroenteritis, chronic urticaria, severe allergic conjunctivitis, and indolent systemic mastocytosis; e.g., Clinicaltrials.gov identifiers NCT03496571, NCT03436797, NCT03379311, and NCT02808793).

\section{Acknowledgement}

We thank Bruce S. Bochner, M.D. and Robert P. Schleimer, $\mathrm{Ph}$.D. for their critical review and feedback on the manuscript, Ingrid Koo, Ph.D. and Alan T. Chang for providing writing support during manuscript development, and Ken Luehrsen, PhD for reagent development. 


\section{Statement of Ethics}

The collection of human tissue was approved by the Vanderbilt University Institutional Review Board (IRB\# 031078 and 010294). All animal use was approved and in accordance with the guidelines of the Animal Care and Use Committees of the University of Massachusetts Medical School, Northwestern University Feinberg School of Medicine, and The Jackson Laboratory.

\section{Disclosure Statement}

Drs. Bradford A. Youngblood, Rustom Falahati, Christopher Bebbington, and Nenad Tomasevic, and Ms. Emily C. Brock and Jessica Bright and Mr. John Leung and Jason Williams are or were employees and have ownership interest in Allakos Inc., outside the submitted work; Drs. Leonard D. Shultz, Dale L. Greiner, and Michael A. Brehm report personal fees from Allakos, during the conduct of the study. Dr. Leonard D. Shultz also reports personal fees from Allakos, outside the submitted work; Drs. Dale L. Greiner and Michael A. Brehm also received grant support and were consultants for The Jackson Laboratory. Dr. Paul J. Bryce has no conflicts of interest to disclose.

\section{Funding Sources}

This study was funded by Allakos, Inc.

\section{Author Contributions}

B.A.Y., E.C.B., R.F., P.J.B., L.D.S., D.L.G., M.A.B., and N.T.: designed the experiments. B.A.Y., E.C.B., R.F., J.L., J.B., J.W., P.J.B., and M.A.B.: conducted the experiments. B.A.Y., E.C.B., R.F., J.L., P.J.B., and M.A.B.: acquired data. B.A.Y., E.C.B., R.F., J.L., P.J.B., and M.A.B.: analyzed data. J.L., J.W., L.D.S., and D.L.G.: provided reagents. B.A.Y., E.C.B., N.T., and C.B.: wrote the manuscript.

\section{References}

1 Hudson SA, Herrmann H, Du J, Cox P, Haddad B, Butler B, et al. Developmental, malignancy-related, and cross-species analysis of eosinophil, mast cell, and basophil siglec- 8 expression. J Clin Immunol. 2011 Dec;31(6): 1045-53.

2 Kikly KK, Bochner BS, Freeman SD, Tan KB, Gallagher KT, D’alessio KJ, et al. Identification of SAF-2, a novel siglec expressed on eosinophils, mast cells, and basophils. J Allergy Clin Immunol. 2000 Jun;105(6 Pt 1):1093100.

3 Floyd H, Ni J, Cornish AL, Zeng Z, Liu D, Carter KC, et al. Siglec-8. A novel eosinophilspecific member of the immunoglobulin superfamily. J Biol Chem. 2000 Jan;275(2): 861-6.

4 Yokoi H, Choi OH, Hubbard W, Lee HS, Canning BJ, Lee HH, et al. Inhibition of FcepsilonRI-dependent mediator release and calcium flux from human mast cells by sialic acidbinding immunoglobulin-like lectin 8 engagement. J Allergy Clin Immunol. 2008 Feb;121(2):499-505.e1.

5 Nutku E, Aizawa H, Hudson SA, Bochner BS. Ligation of Siglec-8: a selective mechanism for induction of human eosinophil apoptosis. Blood. 2003 Jun;101(12):5014-20.

6 Nutku-Bilir E, Hudson SA, Bochner BS. Interleukin-5 priming of human eosinophils alters siglec- 8 mediated apoptosis pathways. Am J Respir Cell Mol Biol. 2008 Jan;38(1): 121-4.

7 Nutku E, Hudson SA, Bochner BS. Mechanism of Siglec-8-induced human eosinophil apoptosis: role of caspases and mitochondrial injury. Biochem Biophys Res Commun. 2005 Oct;336(3):918-24.
$8 \mathrm{Na} H$ J, Hudson SA, Bochner BS. IL-33 enhances Siglec- 8 mediated apoptosis of human eosinophils. Cytokine. 2012 Jan;57(1):169-74.

9 Bryce PJ, Falahati R, Kenney LL, Leung J, Bebbington $\mathrm{C}$, Tomasevic N, et al. Humanized mouse model of mast cell-mediated passive cutaneous anaphylaxis and passive systemic anaphylaxis. J Allergy Clin Immunol. 2016 Sep;138(3):769-79.

10 Ganeshan K, Neilsen CV, Hadsaitong A, Schleimer RP, Luo X, Bryce PJ. Impairing oral tolerance promotes allergy and anaphylaxis: a new murine food allergy model. J Allergy Clin Immunol. 2009 Jan;123(1):231-238.e4.

11 Li XM, Serebrisky D, Lee SY, Huang CK, Bardina $\mathrm{L}$, Schofield BH, et al. A murine model of peanut anaphylaxis: T- and B-cell responses to a major peanut allergen mimic human responses. J Allergy Clin Immunol. 2000 Jul; 106(1 Pt 1):150-8.

12 Shields RL, Lai J, Keck R, O'Connell LY, Hong $\mathrm{K}$, Meng YG, et al. Lack of fucose on human IgG1 N-linked oligosaccharide improves binding to human Fegamma RIII and antibody-dependent cellular toxicity. J Biol Chem. 2002 Jul;277(30):26733-40.

13 Shinkawa T, Nakamura K, Yamane N, ShojiHosaka E, Kanda Y, Sakurada M, et al. The absence of fucose but not the presence of galactose or bisecting $\mathrm{N}$-acetylglucosamine of human IgG1 complex-type oligosaccharides shows the critical role of enhancing antibodydependent cellular cytotoxicity. J Biol Chem. 2003 Jan;278(5):3466-73.

14 Tomasevic N, Luehrsen K, Baer M, Palath V, Martinez D, Williams J, et al. A high affinity recombinant antibody to the human EphA3 receptor with enhanced ADCC activity. Growth Factors. 2014 Dec;32(6):223-35.
15 Fulkerson PC, Rothenberg ME. Targeting eosinophils in allergy, inflammation and beyond. Nat Rev Drug Discov. 2013 Feb;12(2): 117-29.

16 Georas SN, Liu MC, Newman W, Beall LD, Stealey BA, Bochner BS. Altered adhesion molecule expression and endothelial cell activation accompany the recruitment of human granulocytes to the lung after segmental antigen challenge. Am J Respir Cell Mol Biol. 1992 Sep;7(3):261-9.

17 Liu LY, Sedgwick JB, Bates ME, Vrtis RF, Gern JE, Kita H, et al. Decreased expression of membrane IL-5 receptor alpha on human eosinophils: II. IL-5 down-modulates its receptor via a proteinase-mediated process. J Immunol. 2002 Dec;169(11):6459-66.

18 O'Sullivan JA, Carroll DJ, Cao Y, Salicru AN, Bochner BS. Leveraging Siglec-8 endocytic mechanisms to kill human eosinophils and malignant mast cells. J Allergy Clin Immunol. 2018 May;141(5):1774-1785.e7.

19 Robida PA, Puzzovio PG, Pahima H, LeviSchaffer F, Bochner BS. Human eosinophils and mast cells: birds of a feather flock together. Immunol Rev. 2018 Mar;282(1):151-67.

20 Johansson MW, Kelly EA, Nguyen CL, Jarjour NN, Bochner BS. Characterization of Siglec-8 expression on lavage cells after segmental lung allergen challenge. Int Arch Allergy Immunol. 2018;177(1):16-28.

21 Melsen JE, Lugthart G, Lankester AC, Schilham MW. Human Circulating and TissueResident CD56(bright) Natural Killer Cell Populations. Front Immunol. 2016 Jun;7:262.

22 Wang W, Erbe AK, Hank JA, Morris ZS, Sondel PM. NK Cell-Mediated Antibody-Dependent Cellular Cytotoxicity in Cancer Immunotherapy. Front Immunol. 2015 Jul;6:368. 